\title{
A Handy Skin Wound Dressing Prepared by Alginate and Cationic Nanofibrillated Cellulose Derived from Solid Residues of Herbs
}

\author{
Ting Jiang,,${ }^{\mathrm{a}, \mathrm{b},}$ Xiaoyan Feng, ${ }^{\mathrm{c}, \#}$ Rui Xu, ${ }^{\mathrm{c}, \mathrm{d}}$ Sheng Dong, ${ }^{\mathrm{c}}$ Meiyan Wu, ${ }^{\mathrm{c}}$ Xin Zheng, ${ }^{\mathrm{b}, *}$ \\ Weimin $\mathrm{Lu}^{\mathrm{a}}, *$ and $\mathrm{Bin} \mathrm{Li}^{\mathrm{c}, *}$
}

\begin{abstract}
Large amounts of solid residues are generated after extraction of active ingredients from herbs for the production of natural medicine, but the residues have not been well utilized. In this work, cationic nanofibrillated cellulose (CCNF) was prepared from the solid residues of Astragali Radix by etherification and homogenization. The CCNF was mixed with sodium alginate (SA) to create a hydrogel dressing by physical interactions between CCNF and SA without any addition of cross-linker. The CCNFSA dressing exhibited moderate viscosity, good moisture-maintaining property, great antibacterial activities, good cytocompatibility, and clear acceleration of wound healing on rats. Furthermore, this CCNF-SA dressing with nanofibrous structure had moderate air permeability. Therefore, the CCNF-SA hydrogel could be used potentially as a skin wound dressing. Development of cost-effective and bioactive wound dressing materials is of crucial importance to reduce the burden on patients and healthcare systems. Also, this work provides a new strategy for valorization of the solid residues of herbs.
\end{abstract}

Keywords: Cellulose nanofibrils; Alginate; Solid residue of herbs; Skin wound dressing; Wound healing; Cytocompatibility

Contact information: a: Affiliated Hospital of Nanjing University of Chinese Medicine, Nanjing 210029, China; b: Affiliated Qingdao Hiser Hospital of Qingdao University, Qingdao 266033, China; c: Key Laboratory of Bio-Based Materials, Qingdao Institute of Bioenergy and Bioprocess Technology, Chinese Academy of Sciences, Qingdao 266101, China; d: Tianjin Key Laboratory of Pulp and Paper, Tianjin University of Science and Technology, Tianjin 300457, China; *Corresponding authors: zhengxin669@163.com; wmlu@163.com; libin@qibebt.ac.cn; \# The first two authors contributed equally.

\section{INTRODUCTION}

Wounds (chronic or acute wounds, e.g., diabetic ulcers or burns) damage and destroy normal tissue and its functions. Wound infection easily occurs and causes prolonged wound healing if the trauma is mishandled (Naseri-Nosar and Ziora 2018). Thus, a wound dressing is introduced to promote wound healing and prevent bacterial infection (Wang et al. 2020). Skin wound dressing is an important part of the wound care industry with a global market of about \$20.4 billion in 2021 (Homaeigohar and Boccaccini 2020). Therefore, the development of cost-effective and bioactive wound dressing materials is of crucial importance to reduce the burden of patients and healthcare systems.

An ideal wound dressing should have easy fabrication, excellent biocompatibility, sufficient permeability (for exchange of air, water vapor, nutrients, and metabolic waste), good antibacterial ability, nontoxicity, easy use, and non-adherence to the wound (Bajpai et al. 2015). Both natural and synthetic polymers are applied to prepare wound healing materials. Therein, natural materials have aroused increasing attention in wound healing 
due to their good biocompatibility, blood compatibility, biodegradability, and nontoxicity (Naseri-Nosar and Ziora 2018; Homaeigohar and Boccaccini 2020). Many natural polymers including chitosan, pectin, agarose, cellulose, and alginate have been applied in wound protection and dressing (Moeini et al. 2020). Among the rest, cellulose and cellulose derivatives for use in wound dressing has increased recently due to their abundant reserves and renewability (Kanikireddy et al. 2020).

Compared with ordinary cellulose fibers, nanocellulose has unique properties such as nano scale size, high reactive surface, low density, good biodegradability, and excellent moisture maintenance. Nanocellulose includes nanofibrillated cellulose (also called cellulose nanofibril) (CNFs) and cellulose nanocrystals (CNCs) according to their different morphologies (Lv et al. 2019). Compared with the rod-like CNCs, the noodle-like CNFs are more suitable for wound dressing, due to their long fiber length and high aspect ratio, which could endow the dressing nanofibrous structure, mechanical stability, and pliability (Homaeigohar and Boccaccini 2020; Silva et al. 2020). The properties of CNFs are highly dependent on the properties of starting materials and the preparation methods ( $\mathrm{Li}$ et al. 2018). Usually, CNFs are prepared using cellulosic resources as feedstocks (e.g., microcrystalline cellulose, wood pulp, agricultural waste) (Hu et al. 2018). Different functional groups, e.g., ester and carboxyl groups, can be introduced or generated on the surface of nano-cellulose during preparation or surface modification (Li et al. 2015; Lv et al. 2019). Cationic groups, e.g., quaternary ammonium or polyamidoamine dendrimer, can be introduced on the surface of CNFs, and the obtained cationic CNFs (CCNF) can be used as sorbent, antibiotic, or biodegradable flocculants (Zhu et al. 2014; Tehrani and Basiryan 2015; Littunen et al. 2016). Introducing quaternary ammonium salt by etherification is a typical method to prepare CCNF. Quaternary ammonium salts are known for their antibacterial and antifungal properties, which could be the succedaneum to common sanitizer (Bakhshi et al. 2013), and polymers containing cationic quaternary ammonium group are effective as wide-spectrum antibiotics. Hence, it is possible that CCNFs with antibacterial properties could be used in skin wound dressing.

Herbal medicine has played important roles in disease treatment for over 2000 years, particularly in China and Southeast Asia, on account of its relatively smaller side effects and low drug resistance (Ji et al. 2020). To extract active ingredients from herbs for the production of natural medicine, large amounts of cellulosic solid residues are generated. More than 35 million tons of solid residues derived from Traditional Chinese Medicine (TCM) are generated every year in China, and most of the residues are directly buried or incinerated, which causes environmental pollution and wastes cellulosic resources. The cellulosic residues of herbs contain 30 to $50 \%$ cellulose, depending on the plants and extraction process (Chen et al. 2020), and the remaining cellulose in the residues could be used to prepare cellulose-based functional materials or degraded to fermentable saccharides to produce fuels or bio-chemicals (Yu et al. 2018). However, the preparation of CCNFs using cellulosic solid residues of herbs has not been reported in literature.

In this work, CCNF was prepared through quaternization and homogenization of the solid residue of Astragali Radix (AR, the dry root of Astragalus membranaceus, as an example of herbs) after extraction of active ingredients. The obtained CCNF dispersion was mixed with anionic sodium alginate (SA), forming a hydrogel (CCNF-SA), which was expected to be used for wound dressing. Sodium alginate (constituted of 1, 4-linked Dmannuronic acid and L-guluronic acid units) has excellent biocompatibility, good filmforming property and moisture-maintaining property, and it can be used for wound dressing (Pawar and Edgar 2012). However, SA has little antibacterial property, and dry SA film is 
nearly airtight, which is not good for wound healing. Thus, it is speculated that the combination of CCNF and SA would endow the mixture antibacterial property, and the addition of CCNF in anionic SA might reduce the uniformity of the mixture, forming a nanofibrous dressing with improved permeability. Also, tetracycline hydrochloride (TH, as a broad spectrum antibiotic) was loaded in the hydrogel, forming a handy and bioactive wound dressing (CCNF-SA-TH) (Fig. 1). The samples of CCNF, CCNF-SA, and CCNFSA-TH were comprehensively characterized, including antibacterial activities, cytocompatibility, and the in vivo wound healing tests on the skin of rats. In addition, this work provides a new strategy for the valorization of herb extracted residues.

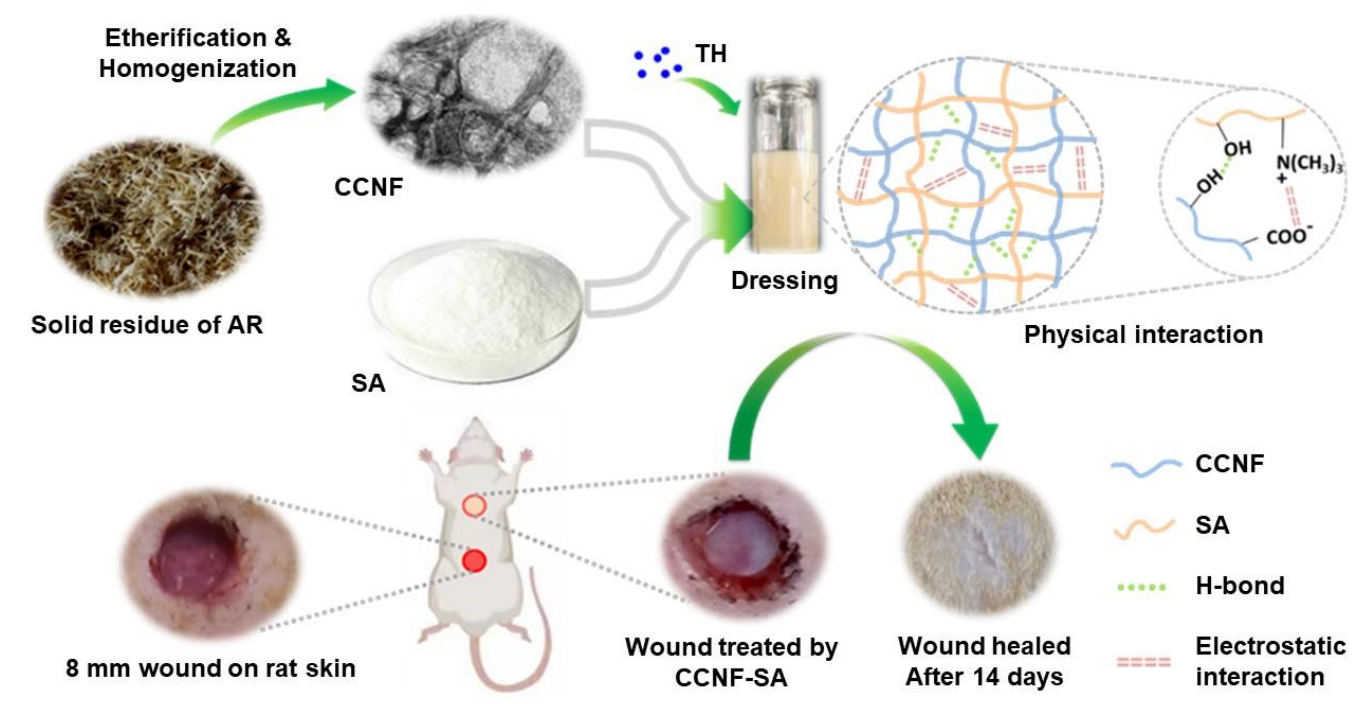

Fig. 1. Schematic illustration of the design and application of CCNF-SA wound dressing on rats

\section{EXPERIMENTAL}

\section{Materials}

The Astragali Radix (AR, the dry root of Astragalus membranaceus (Fisch.) Bge.ver. Mongholicus (Bge.) Hsiao, batch number 1912013) used in this study was purchased from Juyaotang pharmaceutical Co., Ltd., Anguo city, China. The air-dried AR was milled and screened to obtain the particles of 30 to 80 mesh size. To simulate the extraction process of herbal biomass in pharmaceutical factory for the production of natural medicine, the milled AR samples were treated with water-alcohol two-step extraction process according to the National Renewable Energy Laboratory (NREL) analytical procedure (Sluiter et al. 2008). After complete extraction of active ingredients (e.g., saponins, polysaccharides, flavones), the cellulosic solid residues of AR were collected as the starting material for further analysis and the preparation of CCNF. The chemical composition analysis of AR and the solid residues of AR were tested following the standard NREL procedure (Sluiter et al. 2004), and the corresponding chemical compositions are given in Table S1. The cellulose contents of AR and the solid residues of AR were 32 and $73 \%$, respectively. The large increase of the cellulose content of the solid residue of AR was due to the complete removal of extractives during extraction of active compounds from AR.

The reagents of sodium alginate (>99\%, CAS No. 9005-38-3, reference No. 
30164424), glycidyltrimethyl ammonium chloride (GTMAC, $\geq 95 \%$, CAS No. 3033-770, reference No. G-0443804), sodium hydroxide (96\%, CAS No. 1310-73-2, reference No. S111501), tetracycline hydrochloride (TH, 96\%, CAS No. 64-75-5, reference No. T818996), and ethanol ( $\geq 99.5 \%$, CAS No. 64-17-5, reference No. M11172702) were purchased from Sinopham Chemical Reagent Co., Ltd. (Shanghai, China), and used without any purification.

\section{Preparation of Cationic Cellulose Nanofibrils (CCNF)}

The CCNF was prepared via a typical two-step method (alkalization and etherification) using the solid residues of $A R$ as feedstock as previously described, with slight modifications ( $\mathrm{Gu}$ et al. 2020). The solid residue (10 g) of AR was immersed in deionized water with a solids content of $10 \mathrm{wt} \%$ at room temperature $\left(23 \pm 2{ }^{\circ} \mathrm{C}\right)$ for $12 \mathrm{~h}$, and then $\mathrm{NaOH}$ (final concentration $16.7 \mathrm{wt} \%$ ) was added in the suspension. The mixture was continuously stirred $(300 \mathrm{rpm})$ at room temperature $\left(23 \pm 2{ }^{\circ} \mathrm{C}\right)$ for $3 \mathrm{~h}$. After alkalization, the solid residue of $\mathrm{AR}$ was washed with deionized water thoroughly to remove alkali and some dissolved substances (e.g., xylan, lignin). Subsequently, the collected solid residue was mixed with $\mathrm{NaOH}(5 \mathrm{~g})$, GTMAC (10 g), and ethanol (80 mL) in a round-bottom flask for etherification at $50{ }^{\circ} \mathrm{C}$ for $15 \mathrm{~h}$. Upon completion, the solid residues were rinsed with deionized water until neutral $\mathrm{pH}$ was achieved. Finally, the etherified solid residues were dispersed in $700 \mathrm{~mL}$ deionized water, and the suspension was homogenized by a high-pressure homogenizer (Antos Nano Technology Co., Ltd., Suzhou, China) at 650 to 700 bar for $30 \mathrm{~min}$ to obtain the CCNF (0.65 wt\%). The CCNF was concentrated with evaporation to a solids content of $1.4 \mathrm{wt} . \%$. The yield of the resultant CCNF was $50 \pm 2 \%$ based on the dry weight of the original solid residue of AR.

\section{Preparation of CCNF-SA hydrogel Dressing}

The CCNF-SA hydrogel dressing was prepared via physical interaction between CCNF and SA. Briefly, the CCNF $(1.4 \mathrm{wt} \%)$ and SA were mixed at different mass ratios $\left(1: 1,1: 2,1: 3\right.$, and 1:4) and stirred $(600 \mathrm{rpm})$ at room temperature $\left(23 \pm 2{ }^{\circ} \mathrm{C}\right)$ for $1 \mathrm{~h}$ to form uniform hydrogels. The TH was added in the CCNF-SA hydrogel, and the mixture was continuously stirred $(600 \mathrm{rpm})$ at room temperature for $1 \mathrm{~h}$ to get the final homogeneous wound dressing (CCNF-SA-TH). The pure SA was used as control for comparison. The obtained samples were stored at $4{ }^{\circ} \mathrm{C}$.

In real application, the hydrogel dressings will form films after drying, and the air permeability of the formed film will affect the wound healing. Therefore, to simulate the real application and measure the oxygen permeability rates of the formed films, the corresponding film samples of the hydrogels were prepared by the casting method. In brief, $5 \mathrm{~g}$ the aforementioned dressing sample was evenly laid in a glass dish with a diameter of $10 \mathrm{~cm}$. After oven drying at $50{ }^{\circ} \mathrm{C}$ for $6 \mathrm{~h}$, the obtained samples were uniform films with a thickness of approximately $0.03 \mathrm{~mm}$.

\section{Characterization of CCNF and CCNF-SA Wound Dressing}

Because the solid residue of AR was the starting material for the preparation of CCNF, the ATR-FTIR spectra of the freeze-dried CCNF and the solid residue of AR were measured using a Nicolet 6700 FTIR spectrometer (Thermo Fisher Scientific, Waltham, MA, USA) with a wavenumber range of 4000 to $700 \mathrm{~cm}^{-1}$, to verify the successful introduction of cationic groups on the surface of CCNF. The XRD patterns of the freezedried $\mathrm{CCNF}$ and the solid residue of AR were analyzed using an X-ray diffractometer 
(Bruker Discover D8, Karlsruhe, Germany) with Ni-filtered $\mathrm{Cu}$ K $\alpha$ radiation at $40 \mathrm{kV}$ and $40 \mathrm{~mA}$. The range of scattering angle $(2 \theta)$ was from 5 to $60^{\circ}$ with a scan rate of $4^{\circ} / \mathrm{min}$. The crystallinity index (CrI) of the solid residue of AR and CCNF samples were calculated by Segal's method (Segal et al. 1959) with the subtraction of the background of the glass.

The morphology of CCNF was observed with a TEM microscope (Hitachi H-7600, Tokyo, Japan) at an accelerating voltage of $100 \mathrm{kV}$. Before measurement, the diluted CCNF suspension (0.001 wt.\%) was ultrasonically treated $(45 \mathrm{kHz}, 180 \mathrm{~W})$ for $30 \mathrm{~min}$. A droplet of CCNF suspension was deposited on a carbon-supported copper grid and dried at room temperature. Subsequently, the dry samples were dyed with uranyl acetate $(20 \mu \mathrm{L}$, $1.5 \mathrm{wt} . \%$ ) to enhance the contrast of images. The degree of substitution (DS) of quaternary ammonium group of CCNF was calculated based on the content of N (Table S2), which was measured by an elemental analyzer (Vario PYRO, Hanau, Germany), according to the following equation (Zaman et al. 2012),

$$
D S=162 \times N \% /(1400-151.6 \times N \%)
$$

where $N \%$ is the weight percentage of nitrogen in CCNF, 162 is the molecular weight of anhydroglucose unit, and 151.6 is the molecular weight of GTMAC.

The zeta potential of SA, CCNF and CCNF-SA samples was tested with a dynamic light scattering instrument (Zetasizer Nano ZS90, Malvern Instruments, Worcestershire, UK). Before testing, the samples were diluted to $0.01 \mathrm{wt} \%$ and then ultrasonically treated $(45 \mathrm{kHz}, 180 \mathrm{~W})$ for $15 \mathrm{~min}$. Also, the charge densities of dressing samples (with solid content of $1.4 \mathrm{wt} \%$ ) were examined by a Particle Charge Detector (BTG instrument, PCD05, Saffle, Sweden) to determine the stoichiometry of the polyelectrolyte complexes.

The Brookfield viscosity of dressing samples was tested with a Brookfield viscometer (DV2T, LV SPINDLE SET, Brookfield, USA) with 64\# rotor. The dynamic viscosity of SA, CCNF-SA, and CCNF-SA-TH dressing samples was measured with an ARES G2 rheometer (TA Instruments, Surrey, USA) with $50 \mathrm{~mm}$ cone plate geometry at $20{ }^{\circ} \mathrm{C}$. The flow viscosity was tested with the continuously increased shear rate in the range of 0.1 to $1000 \mathrm{~s}^{-1}$.

The morphology of the formed films of dressing samples after oven drying was characterized with a scanning electron microscope (SEM, Hitachi S-4800) at $5.0 \mathrm{kV}$. In the mean time, the wet dressing samples after direct freeze-drying were also characterized by SEM under the same conditions to observe the nanofibrous and porous structure of the resultant dressing, because the structure of the freeze-dried hydrogel dressing was closer to that of wet dressing. All samples were coated with gold under vacuum before observation. In addition, oxygen permeability rates (OPR) of SA, CCNF-SA, and CCNFSA-TH films were measured with an analyzer (Labthink, PERME-OX2/230, Jinan, China) with the $\mathrm{N}_{2}$ and $\mathrm{O}_{2}$ flow rates of $10 \mathrm{~mL} / \mathrm{min}$ at $25^{\circ} \mathrm{C}$.

\section{Antibacterial Properties}

The antibacterial properties of CCNF and CCNF-SA-TH samples were measured by the colony-counting method. Bacillus subtilis (B. subtilis, ASM904v1, Gram positive) and Escherichia coli (E. coli, ASM886v2, Gram negative) were chosen as test organisms, and the pure SA was used as control due to its little antibacterial properties (Zhao et al. 2020). The blank without any test sample was evaluated also. The bacterium was preinoculated with fresh cultures overnight in an incubator $(150 \mathrm{rpm})$ at $37^{\circ} \mathrm{C}$ before the antibacterial test. The cultures of each bacteria were diluted with sterilized broth solution to 1010 times, representing the working bacterial dilution. Next, $5 \mathrm{~g}$ antibacterial agent 
solution (SA, CCNF-SA, or CCNF-SA-TH) was put into a $20 \mathrm{~mL}$ tube containing $5 \mathrm{~mL}$ of the working bacterial dilution. All tubes were shaken at $150 \mathrm{rpm}$ and $37^{\circ} \mathrm{C}$ for $4 \mathrm{~h}$. Next, $10 \mu \mathrm{L}$ of sample solution from the bacterial suspensions was spread onto agar plates. After incubation at $37^{\circ} \mathrm{C}$ for $24 \mathrm{~h}$, the number of colonies formed on the agar plates was counted. The antibacterial efficiencies $(A E)$ of CCNF and CCNF-SA-TH were calculated based on the surviving colonies of the one with SA using the following equation,

$$
A E \%=(A-B) / A \times 100
$$

where $A$ and $B$ are the surviving colonies of SA (control) and the test specimen of CCNFSA or CCNF-SA-TH, respectively.

\section{In vitro Cytocompatibility Evaluation}

The cytocompatibility (reflecting the biocompatibility of dressing materials to normal cells) evaluation of CCNF-SA dressing samples was performed in 96-well tissueculture plate with EA.hy926 cells (Procell CL-0272). The cells were cultured in Dulbecco's modified Eagle medium (DMEM, PM 150210) with the addition of $10 \%$ fetal bovine serum (FBS, 164210-500) and $1 \%$ antibiotics (P/S, PB180120) at $37{ }^{\circ} \mathrm{C}$ and $5 \% \mathrm{CO}_{2}$. Before testing, the CCNF-SA dressing samples were exposed to UV light for $1 \mathrm{~h}$.

To measure the biocompatibility of CCNF-SA dressing samples, $100 \mu \mathrm{L}$ cell suspensions in each well were cultured in 96-well plate at $37{ }^{\circ} \mathrm{C}$ for $24 \mathrm{~h}$. Then, $10 \mu \mathrm{L}$ CCNF-SA, and CCNF-SA-TH dressing samples were added in the corresponding wells. All samples were continuously incubated for 24, 48, and 72 h. Subsequently, CCK-8 solution $(10 \mu \mathrm{L})$ was put in each well, and then all samples were further incubated for another $1 \mathrm{~h}$. Finally, the 96-well medium plate with samples was placed in a microplate reader (BioTek Epoch Z, Winooski, VT, USA) to read the optical density (OD) of each sample at $450 \mathrm{~nm}$. The cell medium without any dressing sample was used as blank. The cell viability percent was calculated by the following equation,

$$
\text { Cell viability }(\%)=\left(\left(A_{s}-A_{b}\right) /\left(A_{c}-A_{b}\right)\right) \times 100
$$

where $A_{s}$ is the absorbance value of the experimental sample well, $A_{c}$ is the absorbance value of the blank well with cell, and $A_{b}$ is the absorbance value of the blank well without cell. All assays were conducted in at least triplicate in the same assay plate, and the average with standard deviation for each sample test was reported.

For the cell LIVE/DEAD fluorescence staining test, the cells were first washed with Dulbecco's phosphate buffer saline after incubation at $37^{\circ} \mathrm{C}$ for $72 \mathrm{~h}$, and the cells were stained by $100 \mu \mathrm{L}$ calcein-AM $(2 \mathrm{mM}) /$ propidium iodide $(4 \mathrm{mM})$ solution at $37{ }^{\circ} \mathrm{C}$ for 30 min. Finally, the cytocompatibility of CCNF-SA based dressing samples were analyzed by a fluorescence microscope (Nexcope, NIB900-FL, Ningbo, China). Calcein-AM was stained within live cells with green fluorescence at $490 \mathrm{~nm}$, and propidium iodide represented dead cells with red fluorescence at $545 \mathrm{~nm}$.

\section{In vivo Wound Healing on Rats}

The in vivo wound healing on rats was conducted according to the laboratory animal administrations rules of China and the guidelines of the National Institutes of Health guide for the care and use of Laboratory animals (NIH Publications No. 8023, revised 1978). The detailed experiment procedures for the in vivo wound healing on rats in this work have been approved by the local ethical committee (Ethical committee of Affiliated Qingdao Hiser Hospital of Qingdao University (Project identification code: 2020HC06LZ001)). 
Each male Wistar rat used in this study was about 250 to $300 \mathrm{~g}$, and all rats were purchased from Beijing Vital River Laboratory Animal Technology Co., Ltd. (China). In a typical experiment, the Wistar rats were first narcotized with pentobarbital sodium ( $1 \mathrm{wt} \%)$. The back hair of rats was barbered carefully with an electric shaver and cleaned with medicinal alcohol. Subsequently, 6 full-thickness circle wounds $(8 \mathrm{~mm})$ were very carefully made on the back of rat by a biopsy punch. Therein, a blank wound without any treatment was used as a control, while SA, CCNF, CCNF-SA, and CCNF-SA-TH dressing samples were applied on the other five wounds. At least five parallel experiments were carried out on each sample. The wounds were covered with a $5 \times 10 \mathrm{~cm}^{2}$ medical gauze to fix the dressing samples, and the corresponding dressings were changed every 3 days. The rats were euthanized after wound healing for 14 days. The wounded skin including dermis and hypodermis were collected and stored in $4 \mathrm{wt} \%$ buffered paraformaldehyde for solidification. After they were embedded in paraffin, the sections of 3 to $5 \mu \mathrm{m}$ (thickness) were stained with hematoxylin-eosin and Masson's trichrome for the observation of tissue appearance and histological analysis, respectively.

The statistics analysis for the antibacterial activity test, in vitro cytocompatibility evaluation and the measurement of collagen fibers of rat skins was performed with GraphPad Prism 8 software (GraphPad, Inc. San Diego, CA, USA) and a one-way ANOVA statistical treatment for the obtained data was conducted.

\section{RESULTS AND DISCUSSION}

\section{Characterization of CCNF}

CCNF were successfully prepared by cationic etherification and homogenization using the solid residues of AR (a solid organic waste of herb extracted residues) as raw material and GTMAC as etherifying agent. The introduction of quaternary ammonium groups was confirmed by ATR-FTIR analysis (Fig. 2a). In the case of the solid residue of $\mathrm{AR}$, the characteristic bands include the broad band at about $3320 \mathrm{~cm}^{-1}$ belonged to the stretching vibration of $\mathrm{O}-\mathrm{H}$, the band at $2920 \mathrm{~cm}^{-1}$ assigned to the stretching vibration of $\mathrm{C}-\mathrm{H}$, the band at $1640 \mathrm{~cm}^{-1}$ originated from the absorbed moisture, and the band at 1030 $\mathrm{cm}^{-1}$ derived from C-O-C stretching vibration in the skeleton of anhydroglucose units (Fei et al. 2018). The same bands were also exhibited in the spectra of CCNF, but the peak value of the broad band relating to hydrogen bonding was shifted from 3320 (the solid residue of AR) to $3360 \mathrm{~cm}^{-1}$ (CCNF) due to the introduction of GTMAC on the surface of CCNF. Additionally, as displayed in inset in Fig. 2a, the relative increase of the band intensity at 1060 and $1163 \mathrm{~cm}^{-1}$ (stretching vibration of ether bond C-O-C) (Li et al. 2019) also confirmed the successful preparation of CCNF. Similar works were also reported previously for the preparation of cationic CNC (Zaman et al. 2012) and cationic microfibrillated cellulose (Zhu et al. 2014).

The XRD patterns of solid residue of AR and CCNF are shown in Fig. 2b. The patterns of the solid residue of AR were similar to those of the native cellulose (cellulose $\mathrm{I} \beta$ ), and the characteristic peaks of solid residue of AR were loaded at $2 \theta=16.6,22.6$, and $34.8^{\circ}$, which are the positions of (110), (200), and (004) crystallographic plane reflections, respectively (Gupta et al. 2013). The characteristic peaks of CCNF were loaded at $2 \theta=$ $12.3,20.1$, and $21.9^{\circ}$, which are the positions of (1-10), (110), and (020) lattice planes, respectively, of the cellulose II crystalline structure. The change of crystalline structure of cellulose was due to the $\mathrm{NaOH}$ treatment for cellulose activation before etherification. In 
this process, cellulose was swelled and recrystallized in an anti-parallel manner to form cellulose II polymorph after washing with water (Goswami et al. 2009). Moreover, the crystallinity index $(\mathrm{CrI})$ of solid residue of AR $(32.9 \%)$ was clearly lower than that of CCNF (67.4\%). The increase of CrI was due to the removal of amorphous components from the solid residue of $\mathrm{AR}$ in the stage of $\mathrm{NaOH}$ treatment before esterification.

The TEM image (Fig. 2c) shows the highly interlaced bundles of CCNF inter-fibrils because of hydrogen bonding (Quinlan et al. 2015) and nanofibrous network structure. The diameters of CCNF ranged from 3 to $9 \mathrm{~nm}$ with an average of $5.6 \pm 1.5 \mathrm{~nm}$ (Fig. 2d), and the length of most CCNFs was above 2 to $5 \mu \mathrm{m}$. Furthermore, the degree of substitution (calculated based on $\mathrm{N}$ content (Table S2)), zeta potential, and charge density of the obtained CCNF were $0.17,+54.8 \pm 2.6 \mathrm{mV}$, and $0.0691 \pm 0.0052 \mathrm{mmol} / \mathrm{g}$, respectively, which also revealed that GTMAC was successfully grafted onto CCNF. In fact, the high zeta potential value (absolute value) can lead to a high stability of CCNF suspension in water phase.
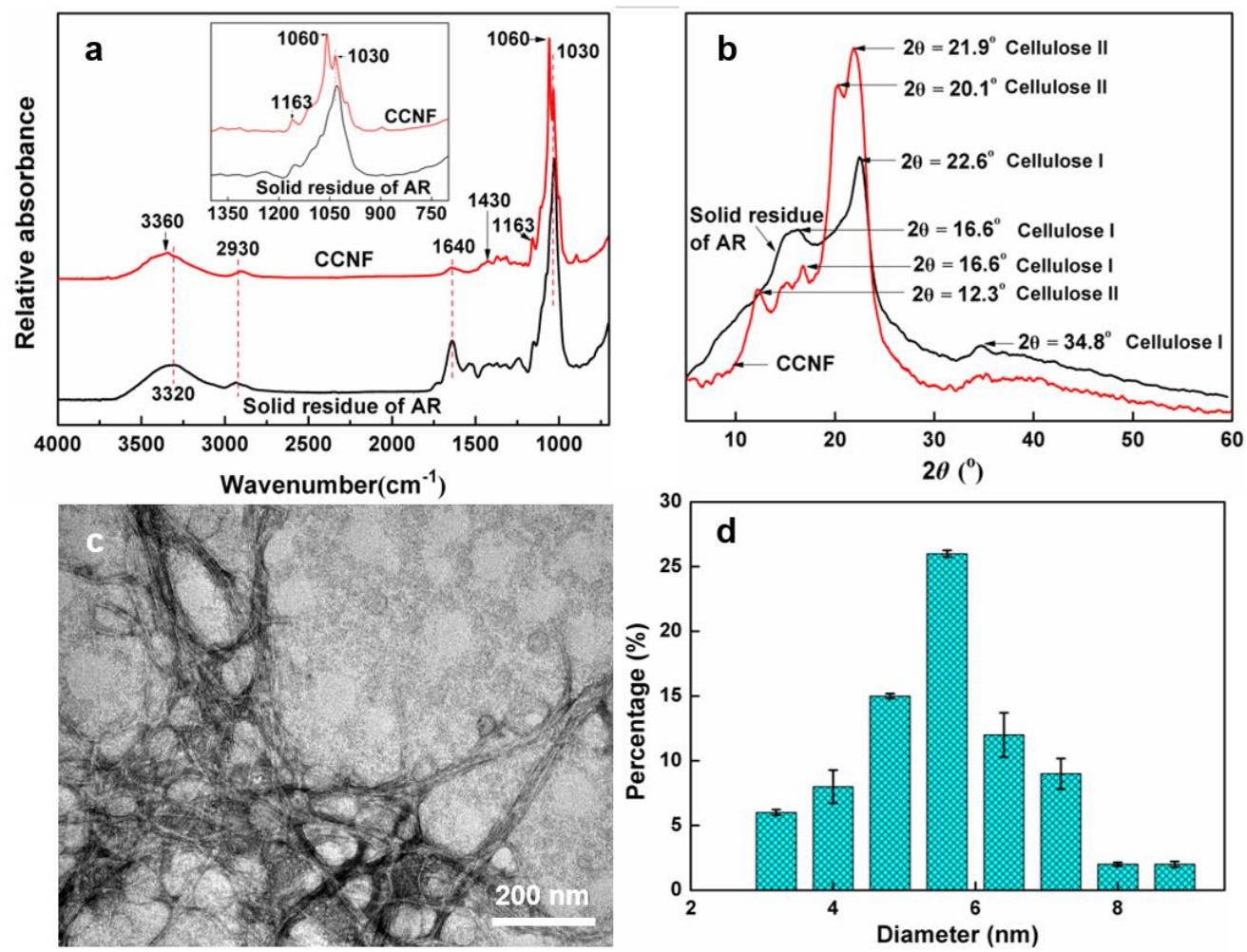

Fig. 2. ATR-FTIR spectra of solid residue of $A R$ and CCNF (a), XRD patterns of solid residue of $A R$ and CCNF (b), TEM image of CCNF (c), and diameter distribution of CCNF (d).

\section{Characterization of CCNF-SA Wound Dressing}

The CCNF-SA wound hydrogel dressing was prepared by mixing of CCNF with $\mathrm{SA}$, and $\mathrm{TH}$ was added in the dressing for comparison (Fig. 1). The hydrogel dressing was formed via the physical interactions (electrostatic interaction, hydrogen bonding, and entanglement) between CCNF and anionic SA without any addition of cross-linker. In this hydrogel dressing, both SA and CCNF have good moisture-maintaining property, which could maintain a moist environment for wound healing. SA also has good film forming property, and CCNF has the expected antibacterial properties. The loaded TH as a broad spectrum antibiotic was expected to enhance antibacterial property and promote wound 
healing.

As shown in Figs. 1, S1 and S2, the appearance of the prepared dressing product is a hydrogel. The viscosity of the CCNF-SA dressing could be tuned by changing the mass ratio of CCNF to SA (charge ratio of CCNF to SA was changed accordingly). As shown in Table 1, with the decrease of the mass ratio of CCNF to SA, the Brookfield viscosity of the CCNF-SA dressing increased. For instance, when the mass ratio of CCNF to SA decreased from 1:2 to 1:4, the Brookfield viscosity of the prepared dressing significantly $(p<0.01)$ increased about 3 times. Polyelectrolyte complexation through electrostatic interactions could play a dominant role in governing the nanostructure of the obtained hydrogel dressing at a relatively lower amount of CCNF. In this case, part of SA was incorporated into the surfaces of CCNF, but the remaining SA with a high absolute value of zeta potential (correspondingly high charge density) could tend to an extended structure, leading to more entanglement of SA chains (Melro et al. 2021). To a relatively small extent, physical entanglement among the fibrous CCNF still existed (as shown in Fig. 3d). Thus, under these physical interactions, a relatively stable network structure of the hydrogel could form. However, the dressing with a high mass ratio of CCNF to SA (1:1) was very easy to flow (due to the lower viscosity). This phenomenon was because higher amounts of CCNF could counteract much negative charges of SA (corresponding charge density decreased to $0.0308 \mathrm{mmol} / \mathrm{g}$, zeta potential of the dressing was only $+0.4 \mathrm{mV}$, and the charge ratio of CCNF to SA in the dressing increased to 1.02 , as presented in Table 1), causing precipitation or contraction of the hydrogel and thus opening up parts of the volume that are mostly water. In this case, the network structure of hydrogel could be broken, causing a much lower viscosity. Hence, the dressing with a high mass ratio of CCNF to SA $(1: 1)$ could not well cover the wound for protection. Moreover, the dressing with a low mass ratio of CCNF to SA (1:4) could generate a thicker film after natural drying, resulting in a relatively lower air permeability (as shown in Table 2), which would not benefit wound healing. Therefore, the more suitable mass ratio of CCNF to SA of the dressing emulsion was between $1: 2$ and 1:3, and the ratio of 1:3 was used for further test.

Table 1. Zeta Potential, Charge Density and Brookfield Viscosity of Dressing Samples with Different Mass Ratios of CCNF to SA

\begin{tabular}{|c|c|c|c|c|}
\hline $\begin{array}{c}\text { Mass Ratio of } \\
\text { CCNF to SA of } \\
\text { dressing }\end{array}$ & $\begin{array}{c}\text { Charge density } \\
(\mathrm{mmol} / \mathrm{g})\end{array}$ & $\begin{array}{c}\text { Charge ratio of } \\
\text { CCNF to SA of } \\
\text { dressing }\end{array}$ & $\begin{array}{c}\text { Zeta Potential } \\
(\mathrm{mV})\end{array}$ & $\begin{array}{c}\text { Brookfield Viscosity } \\
(\text { Pa.s })\end{array}$ \\
\hline $0: 1^{*}$ & $0.0678 \pm 0.0022$ & 0 & $-84.8 \pm 0.9$ & $0.72 \pm 0.02$ \\
\hline $1: 4$ & $0.0624 \pm 0.0048$ & 0.25 & $-67.7 \pm 0.3$ & $9.59 \pm 0.04$ \\
\hline $1: 3$ & $0.0604 \pm 0.003$ & 0.34 & $-59.1 \pm 1.1$ & $6.91 \pm 0.02$ \\
\hline $1: 2$ & $0.053 \pm 0.0039$ & 0.51 & $-49.0 \pm 2.7$ & $2.36 \pm 0.02$ \\
\hline $1: 1$ & $0.0308 \pm 0.0017$ & 1.02 & $+0.4 \pm 0.0$ & $1.21 \pm 0.01$ \\
\hline
\end{tabular}

* Pure SA.

The dynamic rheological properties of the dressing samples of SA, CCNF-SA, and CCNF-SA-TH were measured. Fig. 3a shows that the dressings of SA, CCNF-SA, and CCNF-SA-TH presented a non-Newtonian shear-thinning behavior. Therein, the shear stress of SA, CCNF-SA, and CCNF-SA-TH increased with the increase of shear rate. Correspondingly, the viscosities of SA, CCNF-SA, and CCNF-SA-TH decreased with the increasing of shear rate in the range of 0.1 to $1000 \mathrm{~s}^{-1}$ (Fig. 3b). All the dressing samples had the features of pseudoplastic fluid, which was similar with the paper coatings 
containing nanocellulose (Liu et al. 2017).
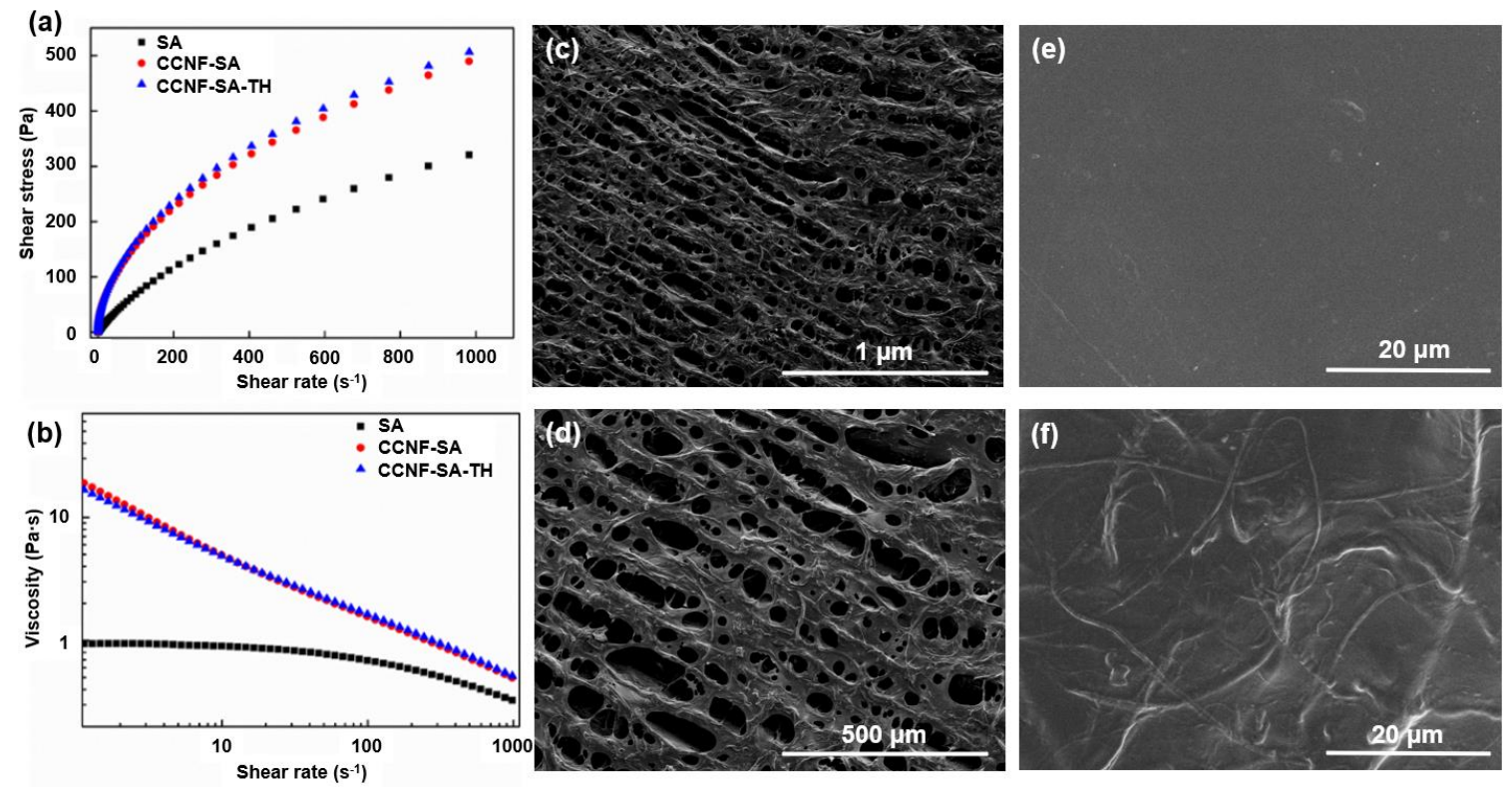

Fig. 3. The dynamic rheological properties of dressing samples (shear rates vs shear stress (a), and shear rates vs viscosity (b)), SEM images of lyophilized CCNF-SA dressing (c and d), and SEM images of the formed films of dressings after oven dried (SA (e), and CCNF-SA (f)) (the mass ratio of CCNF to SA was 1:3).

Figure $3 \mathrm{~b}$ also depicts that, at the same shear rate, the addition of CCNF in SA clearly increased the viscosity of the dressing sample (in agreement with the test of Brookfield viscosity (Table 1)), which was attributed to the strong electrostatic interactions and entanglement between CCNF and anionic SA. Notably, the addition of CCNF in SA could form a nanofibrous and porous structure of the resultant dressing (Figs. 3c and 3d, the structure of the freeze-dried hydrogel dressing was closer to that of wet dressing). Nanofibrous dressing with excellent conformability can better cover and protect the wounds from infection, and nanofibrous meshes with largely exposed surface area and porosity can enhance hemostasis and facilitate interaction with the cells in wound via an extracellular matrix mimicking structure (Homaeigohar and Boccaccini 2020). The nanofibrous structure is also beneficial to drug loading and the permeability of dressing (Homaeigohar and Boccaccini 2020). Interestingly, the addition of TH had no clear impact on the viscosity of the resultant dressing (Fig. 3b). Compared with the pure SA dressing, a relatively higher and moderate viscosity could make CCNF-SA dressing well applied and covered on the wound of the skin for protection.

After oven drying of the dressing samples, the formed films were further characterized. As shown in the SEM images of Fig. 3e, the film of SA was very smooth, homogeneous, and compact. However, the films became coarse and rugged with the addition of CCNF (Fig. 3f), which could increase the permeability of the dressing. Air permeability is an important parameter for wound dressing materials and a moderate air permeability is beneficial for wound healing (Liu et al. 2018). Thus, the oxygen permeability rate (OPR) of the formed films of pure SA, CCNF-SA, and CCNF-SA-TH samples were also tested. As shown in Table 2, the OPR of CCNF-SA film was significantly higher $(p<0.01)$ than that of the pure SA film. The much higher OPR of CCNF-SA film with the combinations of CCNF and anionic SA are consistent with 
polyelectrolyte complexation taking place in a way leading to an irregular and incomplete structure. Compared to the CCNF-SA film, the OPR of the CCNF-SA-TH film was similar, indicating that the addition of $\mathrm{TH}$ in dressing did not affect the air permeability of the CCNF-SA-TH dressing. Furthermore, the air permeability of the CCNF-SA and CCNFSA-TH dressings was comparable with the reported dressing materials (e.g., chitosan/polyethylene glycol fumarate/thymol hydrogel (Koosehgol et al. 2017), which is suitable for wound healing.

Table 2. Oxygen Permeability of Formed Films after Oven Drying

\begin{tabular}{|c|c|c|}
\hline Samples & $\begin{array}{c}\text { Oxygen Permeability } \\
\left(\mathrm{m}^{3} / \mathrm{cm}^{2} \cdot \mathrm{d}\right)\end{array}$ & $\begin{array}{c}\text { Oxygen Transmission Coefficient } \\
\left(\mathrm{cm}^{3} \cdot \mathrm{cm}^{2} / \mathrm{cm}^{2} \cdot \mathrm{S}^{\bullet} \mathrm{cmHg}\right)\end{array}$ \\
\hline SA & $0.0007 \pm 0.0001$ & $7.9 \times 10^{-14} \pm 1.0 \times 10^{-14}$ \\
\hline CCNF-SA $(1: 4)^{*}$ & $1260.6 \pm 376.6$ & $1.7 \times 10^{-7} \pm 0.2 \times 10^{-7}$ \\
\hline${\text { CCNF-SA }(1: 3)^{*}}^{*}$ & $2583.3 \pm 215.0$ & $4.2 \times 10^{-7} \pm 0.4 \times 10^{-7}$ \\
\hline CCNF-SA-TH $(1: 3)^{*}$ & $2029.7 \pm 23.3$ & $3.3 \times 10^{-7} \pm 0.2 \times 10^{-7}$ \\
\hline
\end{tabular}

* The mass ratio of CCNF to SA.

\section{In vitro Antibacterial Activities and Cytocompatibility Evaluation}

As known, avoiding bacterial infection is of vital importance for the promotion of wound healing (Homaeigohar and Boccaccini 2020). Hence, it is also crucial that the wound dressing materials have good antibacterial property. Figure 4a gives the images of bacterium colonies presented in agar plates with SA, CCNF-SA, and CCNF-SA-TH samples, respectively, and Fig. S3 shows the state of blank (without any dressing sample) where the agar plates were fully covered by bacterium colonies. Compared with the control with SA (without antibacterial property (Zhao et al. 2020)), the numbers of bacterium colonies were significantly $(\mathrm{p}<0.01)$ reduced for the one with CCNF-SA, and there were no bacterium colonies left with CCNF-SA-TH sample (Fig. 4a). Correspondingly, the calculated antibacterial rates of CCNF-SA against E. coli and B. subtilis were 90.3 and $84.2 \%$, respectively, while the antibacterial rates of CCNF-SA-TH against E. coli and $B$. subtilis were $100 \%$ (Fig. 4b).
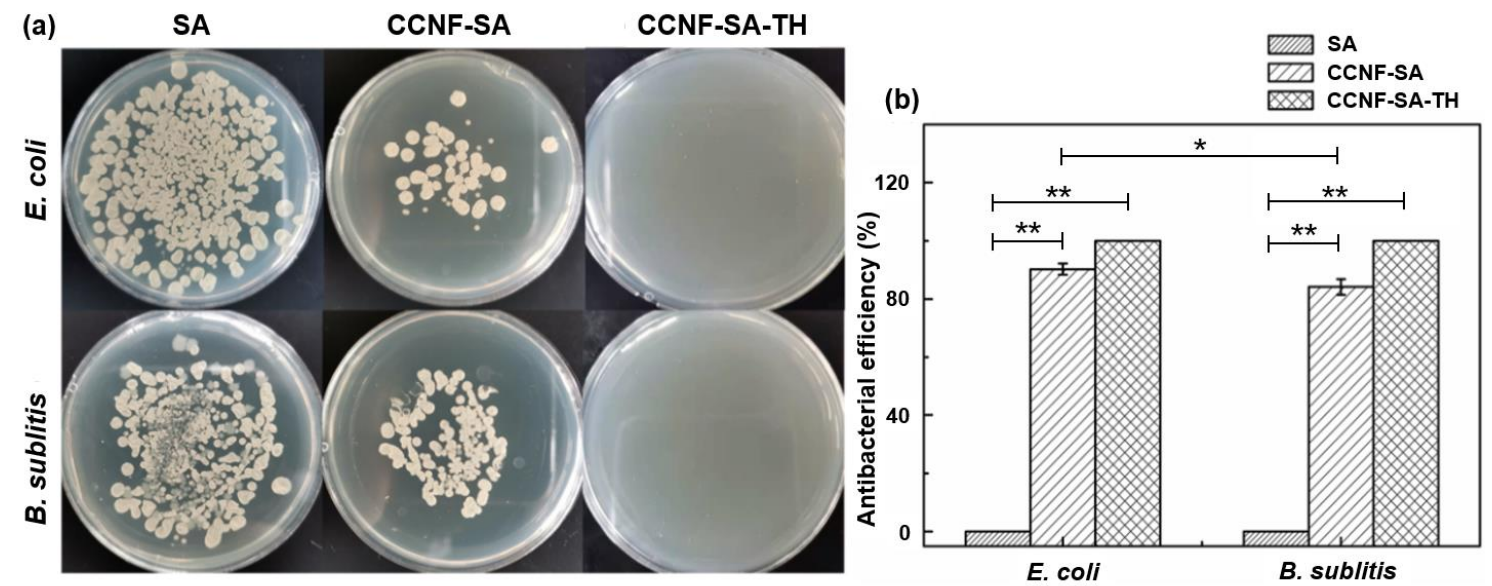

Fig. 4. Antibacterial activities of dressing samples against $E$. coli and $B$. subtilis (a), and the antibacterial rates of dressing samples (b) $\left({ }^{* *}, p<0.01 ;{ }^{*}, p<0.05\right)$.

Quaternary ammonium salt is well known as a broad-spectrum microbicide, and the electrostatic interactions between quaternary ammonium salt and the microbial cell 
membranes could make the hydrophobic components of quaternary ammonium salt lysed the membranes, resulting in the death of bacterial cells (Li et al. 2020). Furthermore, the CCNF-SA-TH sample exhibited more efficient antibacterial performance than CCNF-SA. This is because TH is a broad spectrum antibiotic with low toxicity (Liu et al. 2020). In addition, the bactericidal effect of CCNF-SA on E. coli was relatively higher $(\mathrm{p}<0.05)$ than that on $B$. subtilis, reflecting that the positively charged CCNF has higher electrostatic attraction to the gram-negative E. coli (Fei et al. 2018).

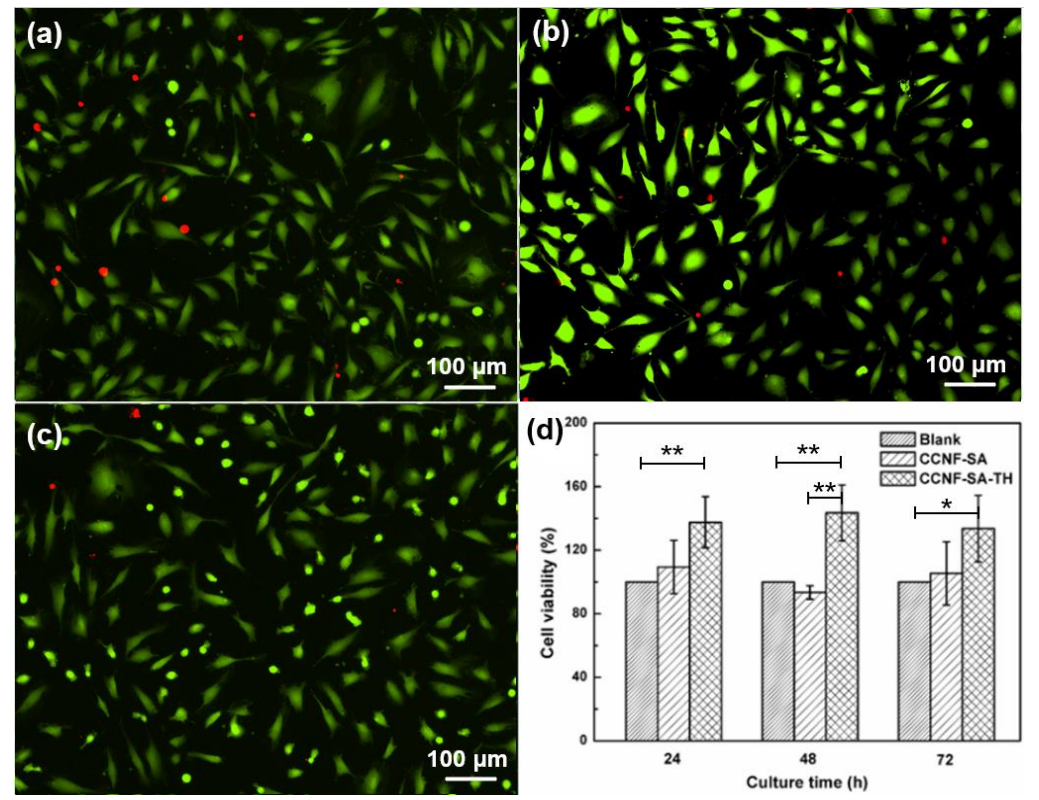

Fig. 5. Fluorescence microscope images of cell co-cultured with dressing samples (Blank (a), CCNF-SA (b), CCNF-SA-TH (c)), and the statistics of cell viability with dressing samples (d) $\left({ }^{* *}, p\right.$ $\left.<0.01 ;{ }^{*}, p<0.05\right)$.

Moreover, good cytocompatibility is a basic requirement for wound dressing materials as well. To assess the cytocompatibility of the CCNF-SA based wound dressing samples, the cell viability was systematically evaluated in vitro using CCK-8 kit for CCNFSA, CCNF-SA-TH with skin cells (EA. hy926 cells). There was no negative effect on cytocompatibility for any CCNF-SA based dressing samples after co-cultured for $72 \mathrm{~h}$, based on the Live/Dead fluorescent staining observation as displayed in Figs. 5a, 5b, and $5 \mathrm{c}$ (the live cells presented green, while the dead cells appeared red under the observation of fluorescence microscope). In addition, the dressing samples improved the cell proliferation with the cell viability reaching 109.4 and $137.6 \%$ for the cells co-cultured for $24 \mathrm{~h}$ with CCNF-SA and CCNF-SA-TH, respectively, compared with the blank (Fig. 5d), and the corresponding values were 105.4 and $133.6 \%$, respectively, after co-culture for 72 h. Therein, the antibiotic (TH) loaded in the CCNF-SA materials showed the highest cell viability for the EA. hy926 cells, suggesting that the dosage of TH ( $5 \mathrm{mg} / \mathrm{g}$-CCNF solution) was safe to use. Therefore, it was concluded that both CCNF-SA and CCNF-SA-TH have good cytocompatibility.

\section{In vivo Wound Healing}

To evaluate the effect of the CCNF-SA based dressing on wound healing on rats, circle dorsal full-thickness cutaneous defects on the skin of Wistar rats were generated. 
Figures 6a and S4 show the representative photographs of the state of the wound healing with the blank, SA, CCNF, CCNF-SA, and CCNF-SA-TH dressing samples after 0, 7, and 14 days' post-surgery.
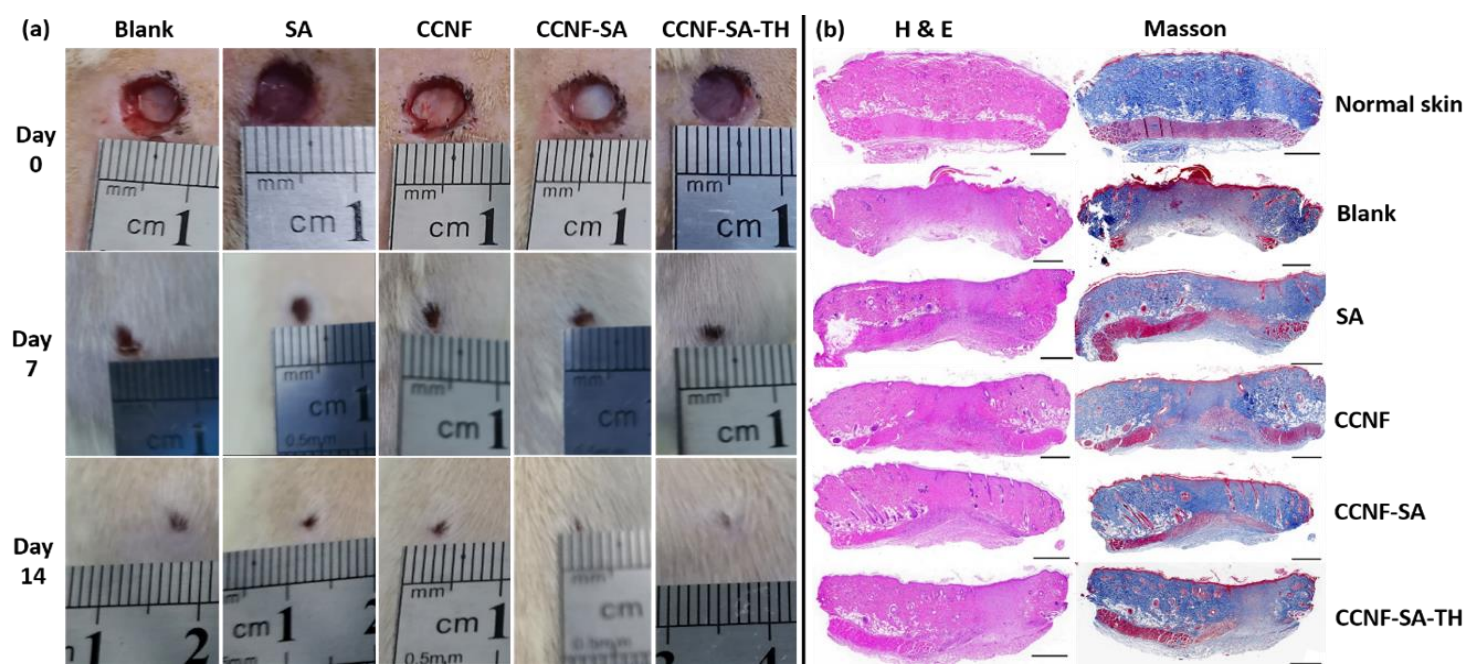

Fig. 6. Pictures of gross appearance of wounds treated with different materials for 0,7 , and 14 days, respectively (a), and the $\mathrm{H}$ and $\mathrm{E}$ staining and Masson staining of wound sections after 14 days of treatment (the scar bar was $1 \mathrm{~mm}$ ) (b).

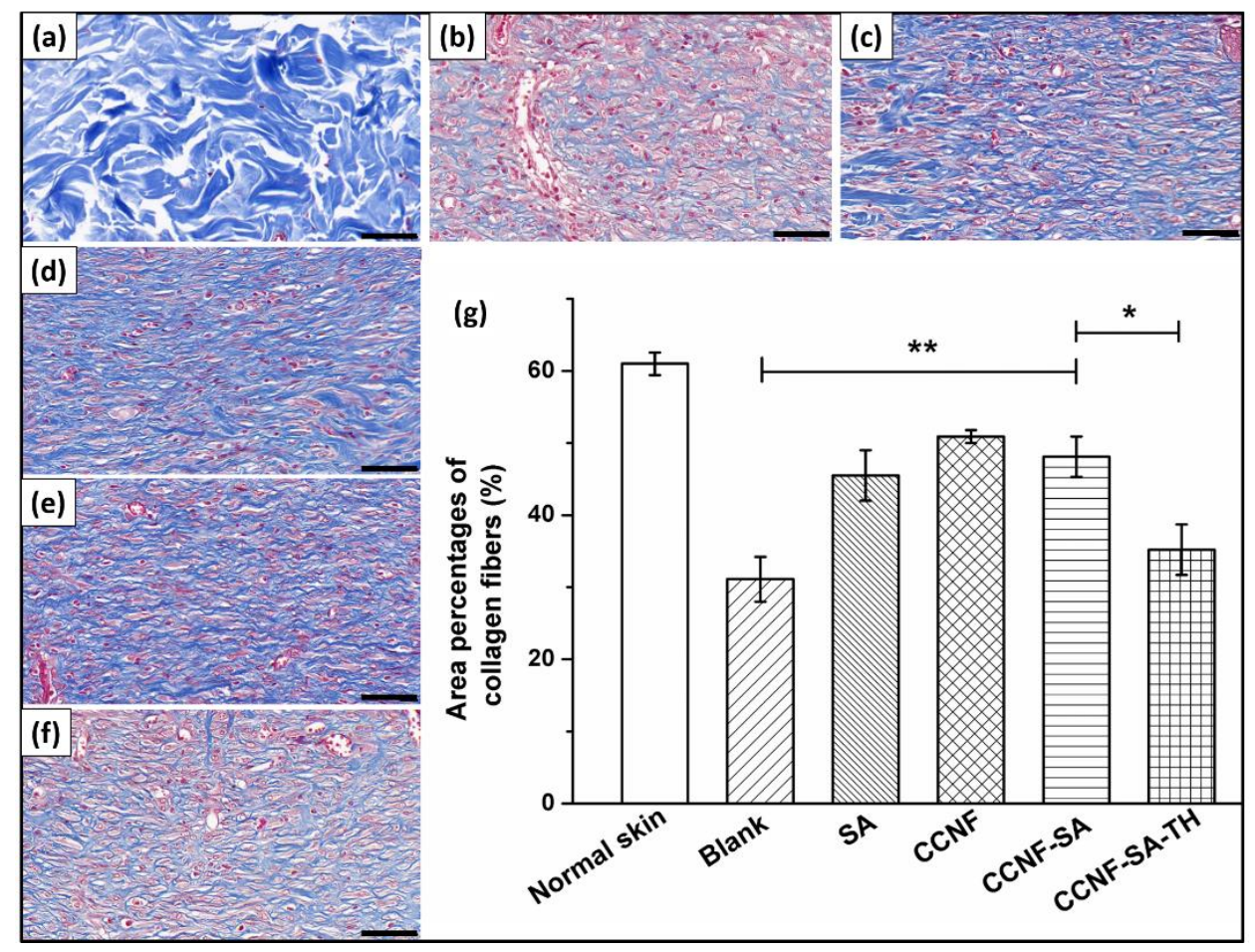

Fig. 7. The images of collagen fibers of wound sections of rats (normal skin (a), blank (b), SA (c), CCNF (d), CCNF-SA (e), and CCNF-SA-TH (f)) after 14 days of treatment (the scar bar was 60 $\mu \mathrm{m})$, and the calculated area percentages of collagen fibers $(g)\left({ }^{* *}, p<0.01 ;{ }^{*}, p<0.05\right)$.

For all wounds, whether they were treated with CCNF-SA based dressing or not, the size of the wound tended to be reduced with the increasing of healing time. After 14 days' treatment, the wounds were almost completely healed in all samples. As expected, 
the CCNF-SA based dressing samples showed excellent wound healing capability compared with the blank.

Histological analysis was conducted after 14 days' healing to further assess the original re-epithelialization and collagen fiber production. Hematoxylin and eosin $(\mathrm{H}$ and E) and Masson's trichrome were used to stain the skin samples adjacent tissues for analysis, respectively. As shown in Fig. 6b, several fibroplasias, necrotic fibers, and more inflammatory cells without complete epidermal layer formation were observed in the blank after 14 days' post-treatment. The wound treatment with SA, CCNF, and CCNF-SA dressing samples led to an almost complete epidermal layer, granulation tissue and hair follicles formation, and mild fibroplasias. The collagenous fibers were thicker and much denser in these samples compared with the blank. A moderate fibroplasias with less inflammation, nearly complete epidermal layer, more hair follicles and neovascularization, as well as longer and tighter adipocyte were seen in the CCNF-SA-TH sample. Intriguingly, the wound treated with CCNF-SA dressing promoted complete epithelization with less inflammation and fibroplasias. More sebaceous glands, hair follicles, neovascularization, and tighter adipocytes appeared with CCNF-SA after 14 days' post-treatment. These results indicated that the CCNF-SA dressings had good curative effect on wound healing.

Collagen maintains the structural integrity of the recovered tissue as the principle extracellular matrix component of the dermis (Moeini et al. 2020). Therefore, collagen formation and distribution in the wounds after 14 days' post-treatment were evaluated by Masson's trichrome staining, and the results are shown in Figs. 7(a)-6(f). In this staining, the blue color presented the relative amount of deposited total collagen and revealed the synthesis process of collagen (Asgarirad et al. 2021). As shown in Fig. 7, the wounds treated with CCNF-SA induced much more mature and regularly arranged collagen compared with the blank. Furthermore, this treatment promoted the rejuvenation of hair follicles and other appendages. Quantitative analysis (Fig. 7g) revealed that the area percentage of collagen fibers with the treatment of CCNF-SA $(48.2 \pm 2.8 \%)$ was significantly higher $(\mathrm{p}<0.01)$ than the blank $(31.1 \pm 3.1 \%)$ after 14 days' post-surgery. Additionally, there was no significant difference of the collagen area between the one with CCNF-SA and the one with SA, or CCNF. The collagen area of the one treated with CCNFSA was significantly larger $(\mathrm{p}<0.05)$ than the one treated with CCNF-SA-TH $(35.2 \pm$ $3.5 \%)$, while there was no significant difference $(\mathrm{p}>0.05)$ between the blank and the one treated with CCNF-SA-TH. These results indicated that the addition of antibiotic (TH) did not accelerate the formation of collagen fibers, although $\mathrm{TH}$ has good antibacterial properties (Fig. 4). The CCNF-SA hydrogel dressing was very suitable as a skin wound dressing material without any impact on the growth of fibroblasts in dermis.

\section{CONCLUSIONS}

1. Cationic nanofibrillated cellulose $(\mathrm{CCNF})$ was first prepared by etherification and the followed homogenization with the solid residues of Astragali Radix (AR) as the starting material. The prepared CCNF with the degree of substitution of 0.17 and zeta potential of $+54.8 \pm 2.6 \mathrm{mV}$ had an average diameter of $5.6 \pm 1.5 \mathrm{~nm}$ and the length above 2 to $5 \mu \mathrm{m}$. 
2. The as-prepared CCNF was mixed with sodium alginate (SA), obtaining a hydrogel dressing by physical interactions (electrostatic interactions and entanglement) between CCNF and SA without addition of cross-linker. The resultant dressing had a moderate viscosity (making it better coverage and protection of wound), nanofibrous structure (increasing oxygen permeability), and good antibacterial property (preventing infection).

3. The cytocompatibility and in vivo wound healing tests verified that the prepared CCNFSA dressing with very good cytocompatibility could accelerate wound healing (particularly for the promotion of the synthesis of collagen fibers) on rat models. Thus, the prepared CCNF-SA hydrogel could be potentially used for skin wound dressing materials.

\section{ACKNOWLEDGMENTS}

The authors are grateful for the support of The Natural Science Foundation of China (No. 31870568), Qihuang Scholars Program of Qihuang Project, Shandong Provincial Natural Science Foundation for Distinguished Young Scholar (China) (No. ZR2019JQ10), Taishan Scholars Program of Shandong Province, and Youth Talents Training Project of Qingdao Traditional Chinese Medicine Hospital (Municipal Hiser Hospital) (No. HCR032019012).

\section{REFERENCES CITED}

Asgarirad, H., Ebrahimnejad, P., Mahjoub, M. A., Jalalian, M., Morad, H., Ataee, R., Hosseini, S. S., and Farmoudeh, A. (2021). "A promising technology for wound healing; in-vitro and in-vivo evaluation of chitosan nano-biocomposite films containing gentamicin," J. Microencapsul. 38(2), 100-107. DOI: 10.1080/02652048.2020.1851789

Bajpai, S. K., Chand, N., Ahuja, S., and Roy, M. K. (2015). "Curcumin/cellulose micro crystals/chitosan films: Water absorption behavior and in vitro cytotoxicity," Int. J. Biol. Macromol. 75, 239-247. DOI: 10.1016/j.ijbiomac.2015.01.038

Bakhshi, H., Yeganeh, H., Mehdipour-Ataei, S., Shokrgozar, M. A., Yari, A., and SaeediEslami, S. N. (2013). "Synthesis and characterization of antibacterial polyurethane coatings from quaternary ammonium salts functionalized soybean oil based polyols," Mater. Sci. Eng. C Mater. Biol. Appl. 33(1), 153-164. DOI:

10.1016/j.msec.2012.08.023

Chen, X., Zhang, Q., Yu, Q., Chen, L., Sun, Y., Wang, Z., and Yuan, Z. (2020).

"Depolymerization of holocellulose from Chinese herb residues by the mixture of lignin-derived deep eutectic solvent with water," Carbohydr. Polym. 248, 116793. DOI: 10.1016/j.carbpol.2020.116793

Fei, P., Liao, L., Meng, J., Cheng, B., Hu, X., and Song, J. (2018). "Non-leaching antibacterial cellulose triacetate reverse osmosis membrane via covalent immobilization of quaternary ammonium cations," Carbohydr. Polym. 181, 11021111. DOI: 10.1016/j.carbpol.2017.11.036

Goswami, P., Blackburn, R.S., El-Dessouky, H.M., Taylor, J., and White, P. (2009). 
"Effect of sodium hydroxide pre-treatment on the optical and structural properties of lyocell," Eur. Polym. J. 45(2), 455-465. DOI: 10.1016/j.eurpolymj.2008.10.030

Gu, H., Gao, X., Zhang, H., Chen, K., and Peng, L. (2020). "Fabrication and characterization of cellulose nanoparticles from maize stalk pith via ultrasonicmediated cationic etherification," Ultrason. Sonochem. 66, 104932. DOI: 10.1016/j.ultsonch.2019.104932

Gupta, P.K., Uniyal, V., and Naithani, S. (2013). "Polymorphic transformation of cellulose I to cellulose II by alkali pretreatment and urea as an additive," Carbohydr. Polym. 94(2), 843-849. DOI: 10.1016/j.carbpol.2013.02.012

Homaeigohar, S., and Boccaccini, A.R. (2020). "Antibacterial biohybrid nanofibers for wound dressings," Acta Biomater, 107, 25-49. DOI: 10.1016/j.actbio.2020.02.022

Hu, L., Du, H., Liu, C., Zhang, Y., Yu, G., Zhang, X., Si, C., Li, B., and Peng, H. (2018). "Comparative evaluation of the efficient conversion of corn husk filament and corn husk powder to valuable materials via a sustainable and clean biorefinery process," ACS. Sustain. Chem. Eng. 7(1), 1327-1336. DOI: 10.1021/acssuschemeng.8b05017

Ji, W., Jiang, T., Sun, Z., Teng, F., Ma, C., Huang, S., and Yan, S. (2020). “The Enhanced pharmacological effects of modified traditional chinese medicine in attenuation of atherosclerosis is driven by modulation of gut microbiota," Front. Pharmacol. 11, 546-589. DOI: 10.3389/fphar.2020.546589

Kanikireddy, V., Varaprasad, K., Jayaramudu, T., Karthikeyan, C., and Sadiku, R. (2020). "Carboxymethyl cellulose-based materials for infection control and wound healing: A review," Int. J. Biol. Macromol. 164, 963-975. DOI: 10.1016/j.ijbiomac.2020.07.160

Koosehgol, S., Ebrahimian-Hosseinabadi, M., Alizadeh, M., and Zamanian, A. (2017). "Preparation and characterization of in situ chitosan/polyethylene glycol fumarate/thymol hydrogel as an effective wound dressing," Mater. Sci. Eng. C. Mater. Biol. Appl. 79, 66-75. DOI: 10.1016/j.msec.2017.05.001

Li, B., Xu, W., Kronlund, D., Eriksson, J.-E., Määttänen, A., Willför, S., and Xu, C. (2018). "Comparable characterization of nanocellulose extracted from bleached softwood and hardwood pulps," Paper and Biomaterials 3, 35-44. DOI:10.1021/ie9011672

Li, B., Xu, W., Kronlund, D., Maattanen, A., Liu, J., Smatt, J.H., Peltonen, J., Willfor, S., $\mathrm{Mu}, \mathrm{X}$., and $\mathrm{Xu}, \mathrm{C}$. (2015). "Cellulose nanocrystals prepared via formic acid hydrolysis followed by TEMPO-mediated oxidation," Carbohydr. Polym. 133, 605612. DOI: 10.1016/j.carbpol.2015.07.033

Li, G., Shang, Y., Wang, Y., Wang, L., Chao, Y., and Qi, Y. (2019). "Reaction mechanism of etherification of rice straw with epichlorohydrin in alkaline medium," Sci. Rep. 9(1), 14307. DOI: 10.1038/s41598-019-50860-3

Li, Z., Wang, S., Yang, X., Liu, H., Shan, Y., Xu, X., Shang, S., and Song, Z. (2020). "Antimicrobial and antifouling coating constructed using rosin acid-based quaternary ammonium salt and N-vinylpyrrolidone via RAFT polymerization," Appl. Surf. Sci. 530, 147193. DOI: 10.1016/j.apsusc.2020.147193

Littunen, K., Snoei de Castro, J., Samoylenko, A., Xu, Q., Quaggin, S., Vainio, S., and Seppälä, J. (2016). "Synthesis of cationized nanofibrillated cellulose and its antimicrobial properties," Eur. Polym. J. 75, 116-124. DOI: 10.1016/j.eurpolymj.2015.12.008

Liu, C., Du, H., Dong, L., Wang, X., Zhang, Y., Yu, G., Li, B., Mu, X., Peng, H., and Liu, H. (2017). "Properties of nanocelluloses and their application as rheology 
modifier in paper coating," Ind. Eng. Chem. Res. 56(29), 8264-8273. DOI: 10.1021/acs.iecr.7b01804

Liu, Y., Sui, Y., Liu, C., Liu, C., Wu, M., Li, B., and Li, Y. (2018). “A physically crosslinked polydopamine/nanocellulose hydrogel as potential versatile vehicles for drug delivery and wound healing," Carbohydr. Polym. 188, 27-36. DOI: 10.1016/j.carbpol.2018.01.093

Liu, Y., Fan, Q., Huo, Y., Liu, C., Li, B., and Li, Y. (2020). "Construction of a mesoporous polydopamine@GO/cellulose nanofibril composite hydrogel with an encapsulation structure for controllable drug release and toxicity shielding," ACS. Appl. Mater. Inter. 12(51), 57410-57420. DOI: 10.1021/acsami.0c15465

Lv, D., Du, H., Che, X., Wu, M., Zhang, Y., Liu, C., Nie, S., Zhang, X., and Li, B. (2019). "Tailored and integrated production of functional cellulose nanocrystals and cellulose nanofibrils via sustainable formic acid hydrolysis: Kinetic study and characterization," ACS. Sustain. Chem. Eng. 7(10), 9449-9463. DOI:

10.1021/acssuschemeng.9b00714

Melro, E., Antunes, F. E., da Silva, G .J., Cruz, I., Ramos, P. E., Carvalho, F., and Alves, L. (2021). "Chitosan films in food applications. Tuning film properties by changing acidic dissolution conditions," Polymers 13, 1. DOI: 10.3390/polym13010001

Moeini, A., Pedram, P., Makvandi, P., Malinconico, M., and Gomez d'Ayala, G. (2020). "Wound healing and antimicrobial effect of active secondary metabolites in chitosanbased wound dressings: A review," Carbohydr. Polym. 233, 115839. DOI: 10.1016/j.carbpol.2020.115839

Naseri-Nosar, M., and Ziora, Z. M. (2018). "Wound dressings from naturally-occurring polymers: A review on homopolysaccharide-based composites," Carbohydr. Polym. 189, 379-398. DOI: 10.1016/j.carbpol.2018.02.003

Pawar, S. N., and Edgar, K. J. (2012). "Alginate derivatization: A review of chemistry, properties and applications," Biomaterials 33(11), 3279-3305. DOI: 10.1016/j.biomaterials.2012.01.007

Quinlan, P. J., Tanvir, A., and Tam, K. C. (2015). “Application of the central composite design to study the flocculation of an anionic azo dye using quaternized cellulose nanofibrils," Carbohydr. Polym. 133, 80-89. DOI: 10.1016/j.carbpol.2015.06.095

Segal, L., Creely, J. J., Martin, A. E., and Conrad, C. M. (1959). “An empirical method for estimating the degree of crystallinity of native cellulose using the X-ray diffractometer," Text. Res. J. 29(10), 786-794. DOI: 10.1177/004051755902901003

Silva, N., Garrido-Pascual, P., Moreirinha, C., Almeida, A., Palomares, T., AlonsoVarona, A., Vilela, C., and Freire, C.S.R. (2020). "Multifunctional nanofibrous patches composed of nanocellulose and lysozyme nanofibers for cutaneous wound healing," Int. J. Biol. Macromol. 165, 1198-1210. DOI:

10.1016/j.ijbiomac.2020.09.249

Sluiter, A., Hames, B., Ruiz, R.O., Scarlata, C., Sluiter, J., and Templeton, D. (2004). "Determination of structural carbohydrates and lignin in biomass," Biomass Anal Technol Team Lab Anal Proced 2011, 1-14.

Sluiter, A., Ruiz, R., Scarlata, C., Sluiter, J., and Templeton, D. (2008). "Determination of Extractives in Biomass; Laboratory Analytical Procedure (LAP) ," National Renewable Energy Laboratory 1-9.

Tehrani, A. D., and Basiryan, A. (2015). "Dendronization of cellulose nanowhisker with cationic hyperbranched dendritic polyamidoamine," Carbohydr. Polym. 120, 46-52. DOI: 10.1016/j.carbpol.2014.12.004 
Wang, T., Liao, Q., Wu, Y., Wang, X., Fu, C., Geng, F., Qu, Y., and Zhang, J. (2020). “A composite hydrogel loading natural polysaccharides derived from Periplaneta americana herbal residue for diabetic wound healing," Int. J. Biol. Macromol. 164, 3846-3857. DOI: 10.1016/j.ijbiomac.2020.08.156

Yu, Q., Zhang, A., Wang, W., Chen, L., Bai, R., Zhuang, X., Wang, Q., Wang, Z., and Yuan, Z. (2018). "Deep eutectic solvents from hemicellulose-derived acids for the cellulosic ethanol refining of Akebia' herbal residues," Bioresour. Technol. 247, 705710. DOI: 10.1016/j.biortech.2017.09.159

Zaman, M., Xiao, H., Chibante, F., and Ni, Y. (2012). "Synthesis and characterization of cationically modified nanocrystalline cellulose," Carbohydr. Polym. 89(1), 163-170. DOI: 10.1016/j.carbpol.2012.02.066

Zhao, W.-Y., Fang, Q.-Q., Wang, X.-F., Wang, X.-W., Zhang, T., Shi, B.-H., Zheng, B., Zhang, D.-D., Hu, Y.-Y., Ma, L., and Tan, W.-Q. (2020). "Chitosan-calcium alginate dressing promotes wound healing: A preliminary study," Wound. Repair. Regen. 28(3), 326-337. DOI: 10.1111/wrr.12789

Zhu, X., Wen, Y., Wang, L., Li, C., Cheng, D., Zhang, H., and Ni, Y. (2014). "Binding of sodium cholate in vitro by cationic microfibrillated cellulose," Ind. Eng. Chem. Res. 53(48), 18508-18513. DOI: 10.1021/ie503909g

Article submitted: May 8, 2021; Peer review completed: June 7, 2021; Revised version received and accepted: July 3, 2021; Published: July 12, 2021.

DOI: 10.15376/biores.16.3.5926-5946 


\section{APPENDIX}

\section{SUPPLEMENTARY INFORMATION}

Table S1. Chemical Composition of AR and Solid Residue of AR

\begin{tabular}{ccc}
\hline Samples & AR & Solid residue of AR \\
\hline Cellulose (\%) & $31.5 \pm 0.1$ & $73.0 \pm 0.3$ \\
Xylan (\%) & $2.8 \pm 0.1$ & $5.0 \pm 0.2$ \\
Araban (\%) & $1.8 \pm 0.1$ & $3.5 \pm 0.1$ \\
Acetyl (\%) & $0.5 \pm 0.1$ & $0.52 \pm 0.1$ \\
Water extractives (\%) & $46.4 \pm 0.3$ & - \\
Alcohol extractives (\%) & $0.9 \pm 0.0$ & - \\
Lignin (\%) & $4.7 \pm 0.1$ & $9.2 \pm 0.2$ \\
Ash (\%) & $2.5 \pm 0.1$ & $0.7 \pm 0.1$ \\
\hline
\end{tabular}

Notes: water extractives are the extractives obtained by hot water extraction; alcohol extractives are the extractives obtained by ethanol extraction; lignin includes acid soluble lignin and acid insoluble lignin.

Table S2. Elemental Analysis of CCNF and Solid Residue of AR

\begin{tabular}{cccccc}
\hline Samples & $\mathbf{N} \%$ & $\mathbf{C} \%$ & $\mathbf{H} \%$ & $\mathbf{O} \%$ & $\mathbf{S} \%$ \\
\hline CCNF & 1.63 & 44.38 & 5.76 & 46.97 & 0.57 \\
$\begin{array}{c}\text { Solid residue } \\
\text { of AR }\end{array}$ & 0.39 & 42.91 & 5.70 & 49.42 & 0.56 \\
\hline
\end{tabular}

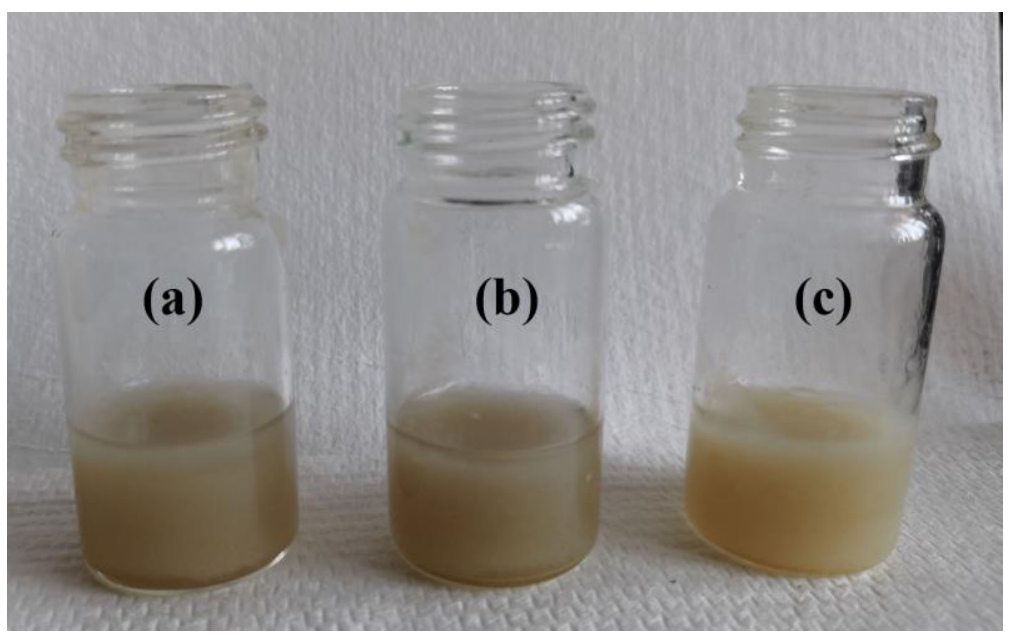

Fig. S1. The photo images of the prepared dressing emulsions (solid content of $5.6 \mathrm{wt} \%$ ) with different mass ratios of CCNF to SA ((a) 1:1, (b) 1:3, (c) 1:4) 


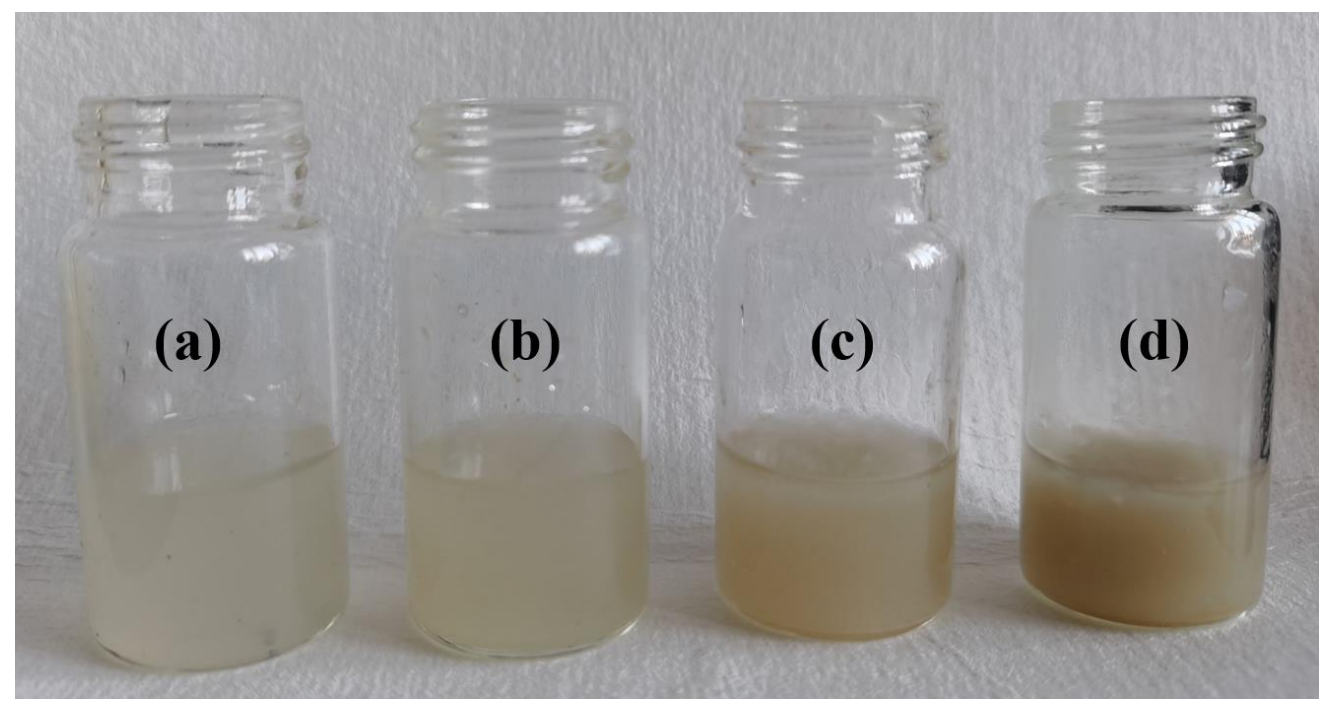

Fig. S2. The photo images of the prepared dressing emulsions (the mass ratio of CCNF to SA is $1: 3$ ) with different solid contents ((a) $0.7 \mathrm{wt} \%$, (b) $1.4 \mathrm{wt} \%$, (c) $2.8 \mathrm{wt} \%$, (d) $5.6 \mathrm{wt} \%$ )

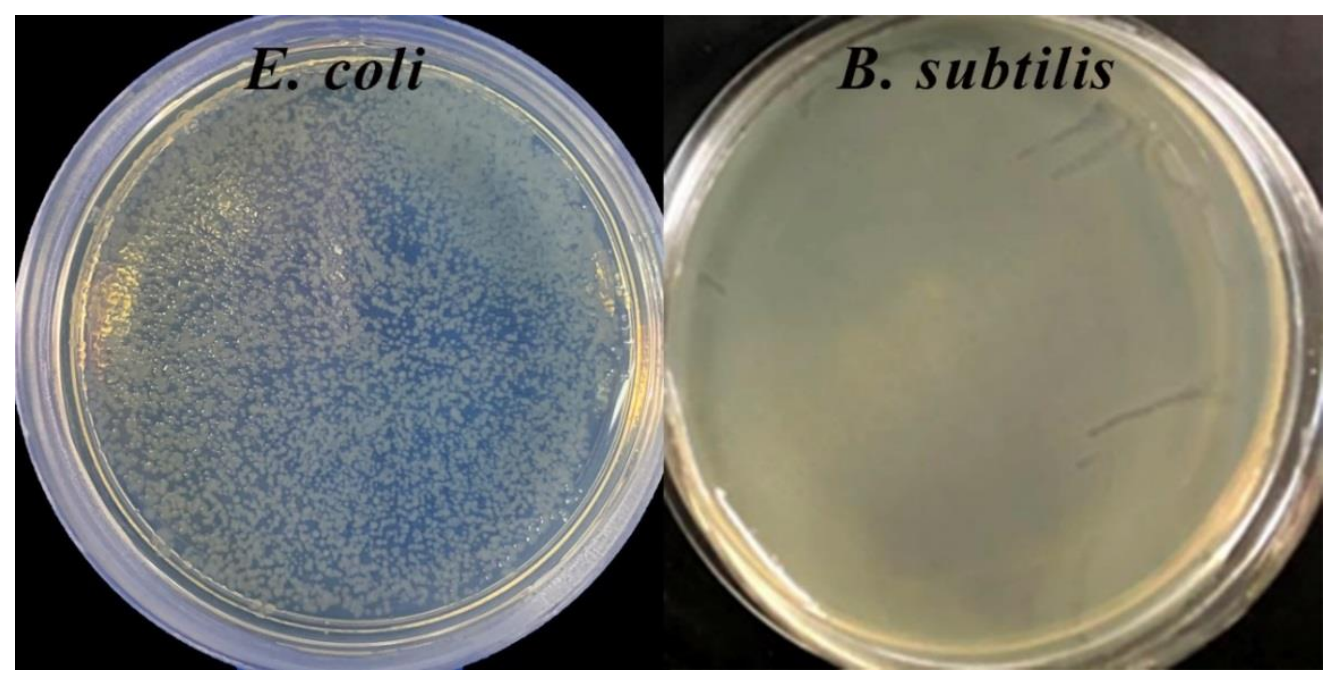

Fig. S3. Blank groups of E. coli and B. subtilis without any test sample 


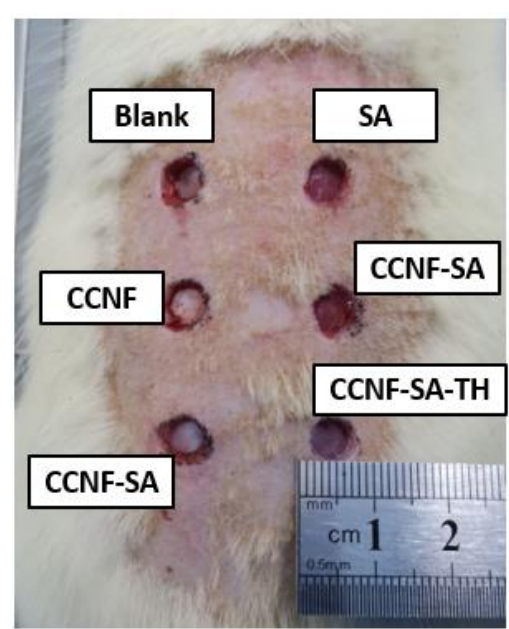

Day 1

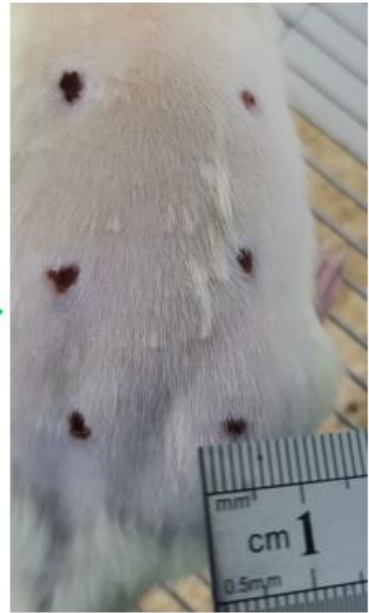

Day 7

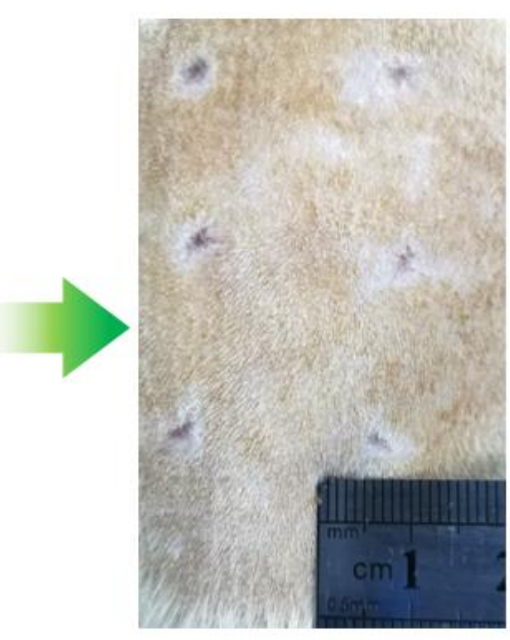

Day 14

Fig. S4. Overview of wound healing process of rats 